ARTICLE

https://doi.org/10.1038/s41467-019-12646-z

\title{
Meiotic crossovers characterized by haplotype- specific chromosome painting in maize
}

Lívia do Vale Martins 1,2,12, Fan Yu1,2,3,12, Hainan Zhao 1,2,12, Tesia Dennison (1) 4, Nick Lauter (10 4,5, Haiyan Wang 1,2, Zuhu Deng ${ }^{3}$, Addie Thompson (1) 6,7, Kassandra Semrau ${ }^{8,11}$, Jean-Marie Rouillard ${ }^{8,9}$, James A. Birchler ${ }^{10} \&$ Jiming Jiang (i) $1,2,7 \star$

Meiotic crossovers (COs) play a critical role in generating genetic variation and maintaining faithful segregation of homologous chromosomes during meiosis. We develop a haplotypespecific fluorescence in situ hybridization (FISH) technique that allows visualization of $\mathrm{COs}$ directly on metaphase chromosomes. Oligonucleotides (oligos) specific to chromosome 10 of maize inbreds B73 and Mo17, respectively, are synthesized and labeled as FISH probes. The parental and recombinant chromosome 10 in $B 73 \times$ Mo17 $F_{1}$ hybrids and $F_{2}$ progenies can be unambiguously identified by haplotype-specific FISH. Analysis of $58 \mathrm{~F}_{2}$ plants reveals lack of $\mathrm{COs}$ in the entire proximal half of chromosome 10. However, we detect COs located in regions very close to the centromere in recombinant inbred lines from an intermated B73 $\mathrm{x}$ Mo17 population, suggesting effective accumulation of COs in recombination-suppressed chromosomal regions through intermating and the potential to generate favorable allelic combinations of genes residing in these regions.

\footnotetext{
${ }^{1}$ Department of Plant Biology, Michigan State University, East Lansing, MI 48824, USA. ${ }^{2}$ Department of Horticulture, Michigan State University, East Lansing, MI 48824, USA. ${ }^{3}$ National Engineering Research Center for Sugarcane, Fujian Agriculture and Forestry University, Fuzhou, China. ${ }^{4}$ Genetics and Genomics Graduate Program, lowa State University, Ames, IA 50011, USA. 5 USDA-ARS Corn Insects and Crop Genetics Research Unit, lowa State University, Ames, IA 50011, USA. ${ }^{6}$ Department of Plant, Soil and Microbial Sciences, Michigan State University, East Lansing, MI 48824, USA. ${ }^{7}$ Michigan State University AgBioResearch, East Lansing, MI 48824, USA. ${ }^{8}$ Arbor Biosciences, Ann Arbor, MI 48103, USA. ${ }^{9}$ Department of Chemical Engineering, University of Michigan, Ann Arbor, MI 48109, USA. ${ }^{10}$ Division of Biological Sciences, University of Missouri, Columbia, MO 65211, USA. ${ }^{11}$ Present address: Department of Natural Sciences, University of Michigan-Dearborn, Dearborn, MI 48128, USA. ${ }^{12}$ These authors contributed equally: Lívia do Vale Martins, Fan Yu, Hainan Zhao. *email: jiangjm@msu.edu
} 
M eiotic recombination, which generates genetic variation via exchange of DNA between homologous parental chromosomes, is essential for plant and animal breeding. Breeders rely on meiotic recombination to create new and favorable combinations of parental alleles. Meiotic recombination is initiated from the formation of double-strand breaks (DSBs) of DNA molecules ${ }^{1}$. DSBs are then repaired through the double Holliday junction or synthesis-dependent strand annealing pathways, which result in meiotic crossovers (COs) or noncrossovers (NCOs), respectively ${ }^{2}$. COs, which create a physical connection of the homologous chromosome pairs, are also essential for faithful chromosome segregation in meiosis. Thus, essentially every chromosome at the first metaphase of meiosis acquires at least one $\mathrm{CO}$, which is known as the obligatory $\mathrm{CO}$ rule $^{3,4}$. The frequency of zero-CO chromosomes is extremely low and is usually much less than $1 \%$ in different organisms ${ }^{4,5}$.

Meiotic COs can be mapped on chromosomes using different methods. COs can be indicated based on the locations of late recombination nodules (RNs) or protein markers associated with COs, such as MLH1, on synaptonemal complexes (SCs) ${ }^{6,7}$. However, it is often difficult or impossible to distinguish individual SCs in the same plant species, which would prevent to map each RN on a specific chromosome. Physical mapping of genetically anchored DNA markers can be used to predict the locations of COs on chromosomes. Physical mapping in plants can be achieved by using cytogenetic stocks ${ }^{8,9}$, or by fluorescence in situ hybridization (FISH) of DNA markers directly on chromosomes $^{10-14}$. However, physical mapping can unveil the relationship between genetical and chromosomal distances of the DNA markers, but does not reveal the exact positions of individual COs. Lastly, genomic in situ hybridization (GISH) can be used to visualize COs derived from homoeologous chromosomes $^{15-17}$. GISH, however, relies on presence of dispersed repeats specific to each chromosone, thus, it can not be used to detect COs from homologous chromosomes.

We develop a FISH technique that allows us to visualize meiotic COs derived from homologous plant chromosomes. We computationally identify large sets of oligonucleotides (oligos) that are specific to chromosome 10 of either maize inbred B73 or Mo17, two highly utilized inbreds that belong to two important opposing heterotic groups ${ }^{18}$ and have been used extensively as parental lines for genetic mapping and heterosis studies ${ }^{19-21}$. The identified oligos are based on presence-absence variation (PAV), single nucleotide polymorphisms (SNPs), and/or insertions and deletions (indels) in chromosome 10 sequences derived from the two inbreds. These oligos are then massively synthesized and labeled as FISH probes. We are able to differentially paint the two copies of parental chromosome 10 in a $\mathrm{B} 73 \times$ Mo17 hybrid using these haplotype-specific FISH probes. We intend to use this FISH technique to detect and quantify meiotic COs derived from the two homologous chromosome 10 in different maize populations.

\section{Results}

Identification of haplotype-specific oligos. Chromosome 10 of maize consists of 150.98 megabases $(\mathrm{Mb})$ of DNA sequences ${ }^{22,23}$. To develop oligo-FISH probes that can be used differentially paint the chromosome 10 from maize inbreds B73 and Mo17, respectively, we used Chorus (https:/github.com/forrestzhang/Chorus) to generate single copy oligos (45 nucleotides (nt)) from the pseudomolecules of chromosome 10 of $\mathrm{B}^{24}$ and $\mathrm{Mo} 17^{23}$. We obtained a total of 175,437 and 174,728 oligos for B73 (B73 oligos) and Mo17 (Mo17 oligos), respectively. We then identified $\mathrm{B} 73$ oligos that are absent in the Mo17 genome and vice versa (see Methods section), which were named PAV oligos. B73 oligos that contain mismatches and/or indels to the Mo17 chromosome
10 sequence, and vice versa, were defined as SNP oligos. We only retained oligos with mismatches and/or indels located between 10-35 bp within each oligo. SNP oligos were identified in pairs (see Methods section), one for B73 and one for Mo17. We calculated the $\Delta \mathrm{Tm}$ between the B73 and Mo17 oligos of each pair using primer $3^{25,26}$. Oligo pairs with $\Delta \mathrm{Tm}>5^{\circ} \mathrm{C}$ were discarded to avoid hybridization bias towards one variant.

Chromosome painting using haplotype-specific oligo probes. We identified 6251 and 5506 PAV oligos specific to B73 and Mo17, respectively. These two sets of oligos were synthesized as two oligo pools. The two synthesized pools of oligos were then amplified, labeled, and hybridized to the metaphase chromosomes prepared from a B73 $\times$ Mo17 hybrid plant. Each pool of oligos generated distinct FISH signals highly specific to the chromosome 10 derived from B73 (red) or Mo17 (green) (Fig. 1a). The B73 PAV probe generated only minimal cross hybridization to chromosome 10 from Mo17, and vice versa. However, the Mo17 probe hybridized to the 45S ribosomal RNA gene region on chromosome 6 from both inbreds (Fig. 1a). This cross hybridization was found to be caused by three oligos that shared $79-88 \%$ sequence similarity with the $45 \mathrm{~S}$ rRNA genes. These three oligos were not detected by the oligo selection software.

We identified 4353 pairs of oligos that are differentiated by 5 or more SNPs between the B73 and Mo17 chromosome 10 sequences. These two sets of oligos generated strong signals on the respective chromosome 10 from B73 or Mo17. We observed weak but visible cross hybridization of the B73 probe to the Mo17 chromosome, and vice versa (Fig. 1b). Similarly, we identified 3894,6506 , and 19,885 pairs of oligos that are differentiated by 3-4 SNPs, 2 SNPs, and 1 SNP, respectively, between the B73 and Mo17 chromosome 10 sequences. Each SNP probe generated stronger signals on the respective chromosome 10 than on the other copy of chromosome 10 in the hybrid. However, the cross hybridization signals became stronger as the number of SNP decreased (Fig. 1). We then used different combinations of these five pairs of oligo pools. The combined pools of oligos with PAV, $\geq 5$ SNPs, and 3-4 SNPs produced the best contrast of haplotype-specific FISH signals (Fig. 2b, c). These two probe pools, thereafter named hapB (haplotype B73, red) and hapM (haplotype Mo17, green), and were used in all future FISH experiments.

Probes hapB and hapM contain 14,498 and 13,753 oligos, respectively. These oligos are not uniformly distributed on chromosome 10 (Fig. 2a). The chromosomal region spanning $42-54 \mathrm{Mb}$, which includes the centromere ${ }^{27}$, contained only 27 and 39 oligos in B73 and Mo17, respectively. Similarly, only 74 (115) oligos were identified in the first $2 \mathrm{Mb}$ on the short arm and 19 (0) oligos identified in the final $1 \mathrm{Mb}$ on the long arm of B73 (Mo17) (Supplementary Data 1). Thus, these regions showed weak or undetectable FISH signals (Fig. 2c).

Meiotic COs revealed by chromosome painting. We wanted to exploit the potential of oligo-FISH using hapB and hapM to detect meiotic COs derived from the homologous chromosome 10 from $\mathrm{B} 73$ and Mo17. We produced $\mathrm{F}_{2}$ seeds by pollinating sibling $\mathrm{B} 73 \times$ Mo17 hybrid plants. Each $\mathrm{F}_{2}$ plant contains two copies of chromosome 10, with each homolog classified as parental or recombinant (Fig. 3). We performed oligo-FISH experiments on somatic metaphase chromosomes from $58 \mathrm{~F}_{2}$ plants (BM1-BM58), resulting in the analysis of 116 copies of chromosome 10. These chromosomes can be cataloged as eight different types based on the positions of chromosomal exchanges (Fig. 3c). We identified at least one unambiguous B73-Mo17 

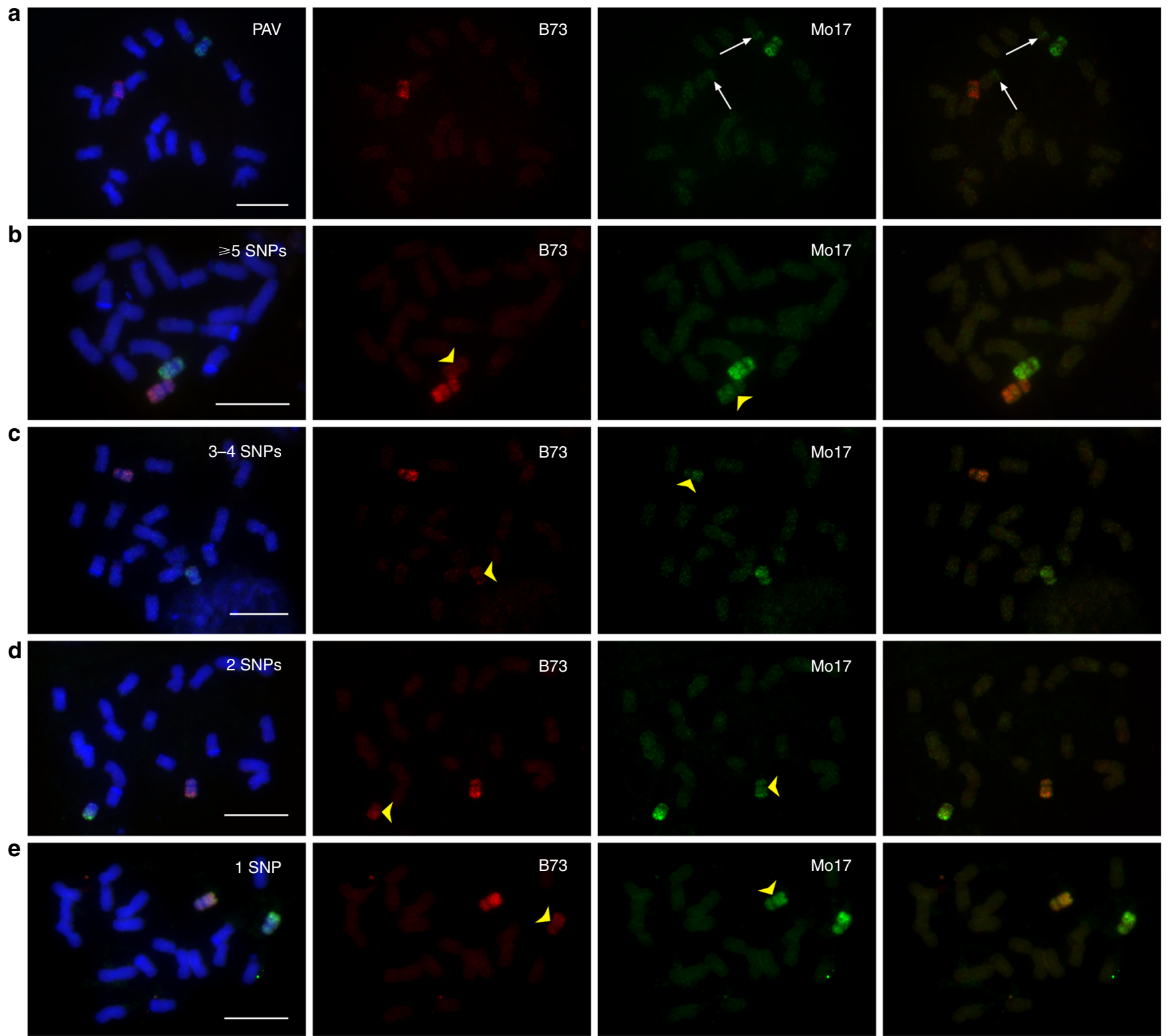

Fig. 1 Development of maize chromosome 10 oligo-FISH probes specific to inbreds B73 or Mo17. a Two oligo-FISH probes based on presence-absence variation. b Two oligo-FISH probes based on 5 or more single nucleotide polymorphisms (SNPs). c Two oligo-FISH probes based on 3 or 4 SNPs. d Two oligo-FISH probes based on 2 SNPs. e Two oligo-FISH probes based on 1 SNP. Oligo-FISH probes specific to the B73 haplotype were detected in red color. Oligo-FISH probes specific to the Mo17 haplotype were detected in green color. Images in first column: Complete metaphase cells hybridized with the two FISH probes; Images in the second column: digitally separated red FISH signals derived from the B73-specific probes; Images in the third column: digitally separated green FISH signals derived from the Mo17-specific probes; Images in the forth column: merged FISH signals derived from both B73 and Mo17. Arrows indicate the cross-hybridization signals at the 45 rDNA region associated with chromosome 6 . Yellow arrowheads indicate the cross-hybridization signals from B73-specific probes to Mo17 chromosome 10, and vice versa. Bars $=10 \mu \mathrm{m}$. The original gray-scale images used to generate the five color images in the first column of all panels of Fig. 1 are provided as a Source Data file

chromosomal exchange on $50(43 \%)$ of the 116 chromosomes (Supplementary Data 2), including 6 chromosomes with an exchange on both arms (Fig. 3a). A chromosome 10 with three or more COs was not identified in the analysis.

We measured the distance $(\mu \mathrm{m})$ from each homologous chromosome exchange position (EP) to the telomere of the respective chromosome arm. This distance was divided by the total length of the respective chromosome arm to calculate the FLA (Fractional Length of the Arm) (see Methods section), which was used to map the position of each EP on the arm (Fig. 4). For example, if the FLA of an EP is 42.5 on the long arm, the distance from this EP to the telomere is $42.5 \%$ of the long arm. We divided the short and long arms of the chromosome into 100 intervals, from 0 at the telomere to 100 at the centromere. Each EP was then mapped to one of these intervals (Fig. 4). Most EPs were located within intervals 20-40 on the short arm and intervals 10-55 on the long arm (Fig. 4). Thus, no EP was observed within nearly half of the chromosome 10 flanking the centromere (Fig. 4). In addition, double COs in the same arm were rare and were found only in one of the 116 copies of chromosome 10 analyzed (Fig. 3b).

Validation of crossovers by genomic sequencing. Recombinant or parental copies of chromosome 10 were unambiguously 

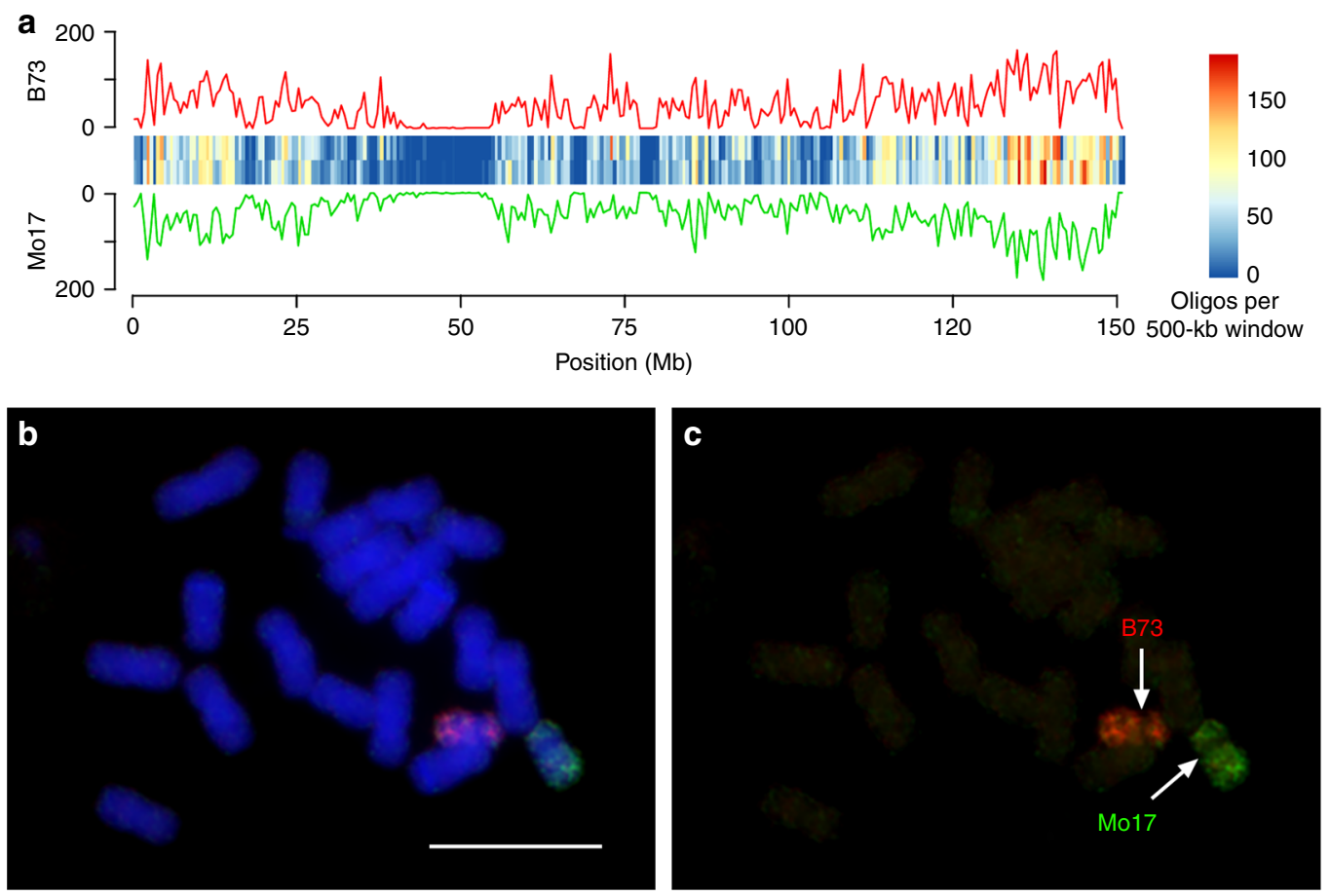

Fig. 2 Development of hapB and hapM probes. a Distribution of 14,498 oligos (red) of probe hapB and 13,753 oligos (green) of probe hapM on maize chromosome 10. The chromosome was divided into 500-kb windows and number of oligos was calculated for each window. The distribution of the number of oligos is shown in the line plot and heatmap. $y$-axis is the number of oligos in each 500-kb window. $\mathbf{b}$ Oligo-FISH on metaphase chromosomes from a B73 $\times$ Mo17 hybrid using hapB (red) and hapM (green). Bar $=10 \mu \mathrm{m}$. c The oligo-FISH signals were digitally separated from panel (b). The original grayscale images used to generate the color image in Fig. $2 b$ are provided as a Source Data file

identified in 54 of the $58 \mathrm{~F}_{2}$ plants analyzed. In the remaining four $\mathrm{F}_{2}$ plants (BM5, BM33, BM34, BM48) (Supplementary Data 2), we observed putative recombinant chromosome(s) with faint FISH signals that were inconsistently observed in the metaphase cells analyzed (Fig. 5). We conducted genomic sequencing of these four plants to validate the inconsistent identification of the COs. In addition, no COs were identified in 18 of 58 (31\%) $\mathrm{F}_{2}$ plants. We suspected that $\mathrm{CO}(\mathrm{s})$ may occur at the distal regions on chromosome 10 in these plants. These COs may not be detectable by FISH due to the lack of a sufficient number of oligos selected from the distal regions (Fig. 2a, Supplementary Data 1). Therefore, we also included six $\mathrm{F}_{2}$ plants (BM8, BM12, BM19, BM21, BM31, BM42), which did not have visible COs, in the sequencing analysis. These 10 plants included 13 copies of chromosome 10 without a visible $\mathrm{CO}$. We generated an average of $2.41 \times$ coverage of $150 \mathrm{bp}$ paired-end Illumina sequences from each of the 10 plants for the analysis.

BM5 contained two copies of Mo17-derived chromosome 10. A CO was detected on the short arm of one chromosome 10 in 8 of 25 (32\%) metaphase cells analyzed (Fig. 5). Sequencing analysis showed that one chromosome 10 has a $\mathrm{CO}$ at $5.04 \mathrm{Mb}$ (Fig. 5). Thus, the oligo-FISH result was supported by the sequencing data. Similarly, BM33 also contained two copies of Mo17-derived chromosome 10. FISH detected a CO on the short arm of one chromosome 10 in 11 of 19 (58\%) metaphase cells analyzed. Sequencing analysis revealed a CO at $6.45 \mathrm{Mb}$ on the short arm of one chromosome (Fig. 5), thereby supporting the $\mathrm{CO}$ observed with oligo-FISH.

BM34 contained two copies of Mo17-derived chromosome 10. We observed hapB FISH signals at the distal regions of the long arms of both chromosome 10 homologs in 6 of 29 (21\%) metaphase cells analyzed (Fig. 5). Sequencing analysis revealed a single $\mathrm{CO}$ on the long arm of each chromosome 10 homolog: at 145.73 $\mathrm{Mb}$ (5.25 Mb away from the end of the long arm telomere) and $148.93 \mathrm{Mb}(2.06 \mathrm{Mb}$ away from the long arm telomere), respectively (Fig. 5). Thus, the sequencing data confirmed the locations of COs on the long arms of both chromosomes.

BM48 contained one B73-derived and one Mo17-derived chromosome 10 . We observed weak hapB FISH signals on the Mo17-derived chromosome 10, and weak hapM FISH signals on the B73-derived chromosome 10, but only in 35\% (9/26) and $8 \%$ $(2 / 26)$ of the metaphase cells, respectively. Sequencing analysis identified a duplicated B73 fragment between $3.86 \mathrm{Mb}$ and 5.42 $\mathrm{Mb}$ on the short arm (Fig. 5). This fragment was possibly resulted from a putative double COs occurred at $3.86 \mathrm{Mb}$ and $5.42 \mathrm{Mb}$, respectively, or two single COs, one at $3.86 \mathrm{Mb}$ on the $\mathrm{B} 73$ derived chromosome 10 , and one at $5.42 \mathrm{Mb}$ on the Mo17derived chromosome 10 (Fig. 5).

COs were not detected by oligo-FISH in BM8, BM12, BM19, and BM21. However, a single CO was discovered on one copy of chromosome 10 in each of these four lines by sequence analysis. The EPs of these four COs were $5.53 \mathrm{Mb}, 2.37 \mathrm{Mb}, 1.69 \mathrm{Mb}$, and $1.74 \mathrm{Mb}$ away from the end of the chromosome, respectively (Fig. 5). COs were not detected in BM31 and BM42 using oligoFISH or sequencing analysis (Fig. 5).

In summary, genomic sequencing of $10 \mathrm{~F}_{2}$ plants revealed COs in 9 of the 20 copies of chromosome 10, including 4 copies of recombinant chromosome 10 that were FISH negative. No COs were detected in BM31 and BM42 (Fig. 5). The comparative analysis showed that COs cannot be reliably detected by the hapB and hapM probes if the EP is located $<5 \mathrm{Mb}$ away from the telomere of a chromosome arm, which contain only $\sim 600$ oligos within each probe (Supplementary Data 1).

Characterization of recombinant inbred lines. B73 and Mo17 have been popular parental lines used in genetic mapping of maize. An intermated $\mathrm{B} 73 \times$ Mo17 recombinant inbred line 


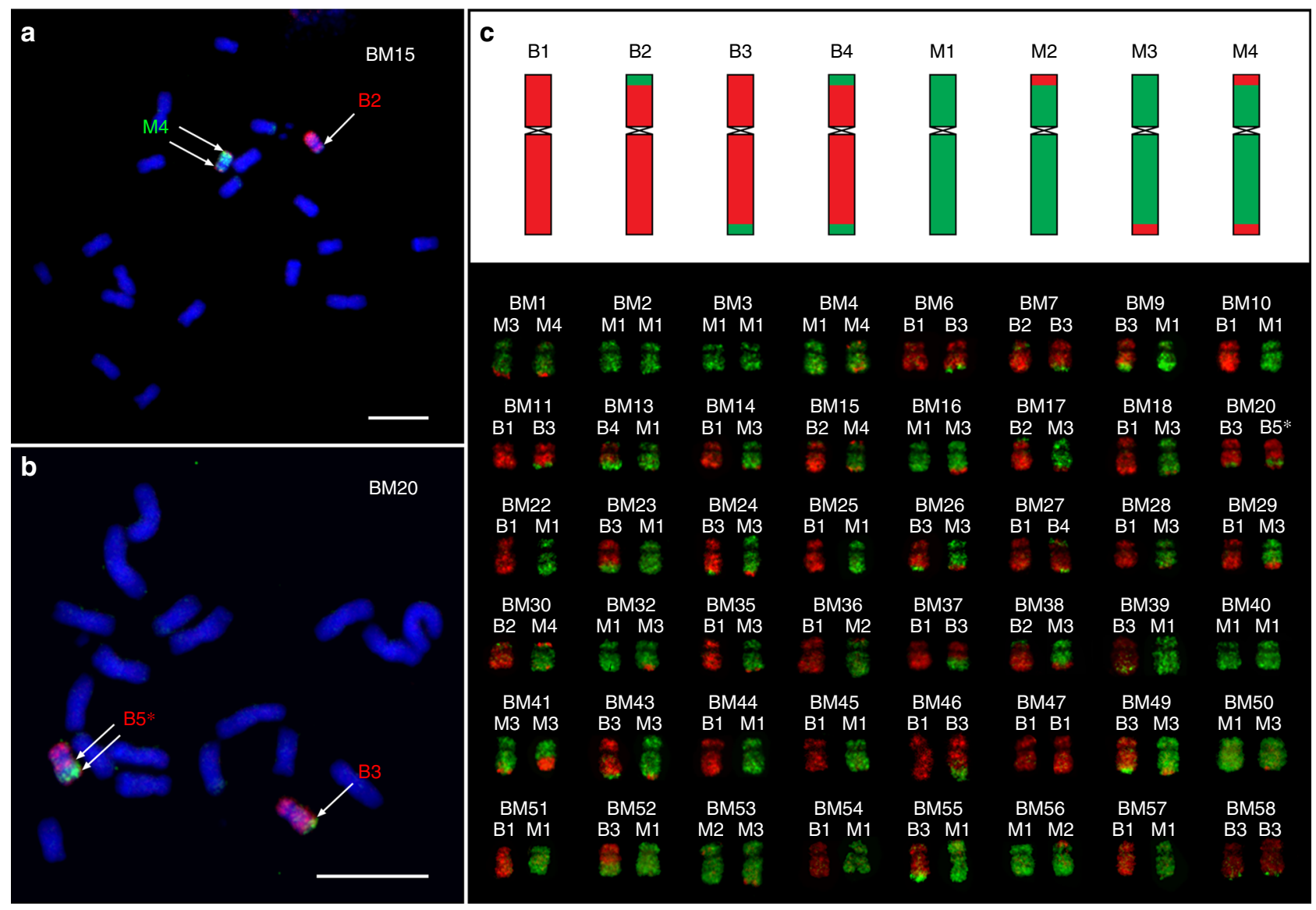

Fig. 3 Crossovers between B73 and Mo17 chromosome 10 revealed by oligo-FISH mapping using probes hapB and hapM. a Oligo-FISH mapping of B73 $\times$ Mo17 $F_{2}$ plant BM15. Probes hapB and hapM are shown in red and green, respectively. The single arrow identifies a single chromosomal exchange position (EP) on the B2-classified chromosome. Double arrows point to the two chromosomal EPs on the M4-classified chromosome. Bar $=10 \mu \mathrm{m}$. $\mathbf{b}$ Oligo-FISH mapping of B73 $\times$ Mo17 F plant BM20. The single arrow points a single EP on the B3-classified chromosome. Double arrows point to the two EPs of double crossovers (COs) on the B5*-classified chromosome. Bar $=10 \mu \mathrm{m}$. c Upper panel: diagrams of the 8 types of parental or recombinant chromosomes identified in $F_{2}$ plants. $B$ indicates B73; $M$ indicates Mo17. Lower panel, representative images of the two copies of chromosomes 10 in $48 F_{2}$ plants. One copy of chromosome 10 in BM20 is marked as B5*, which is the only chromosome that does not belong to any of the eight types listed in the upper panel. The original gray-scale images used to generate the color images in Figs. $3 a$, b are provided as a Source Data file

(IBMRIL) population was developed by randomly intermating plants for four generations following the $\mathrm{F}_{2}$ generation ${ }^{28}$. The increased recombination in this population has resulted in a nearly 4 -fold increase in the genetic map distance compared with conventional nonintermated RIL populations ${ }^{29}$. Thus, chromosomes in IBMRIL are expected to contain four times of more COs compared to the $\mathrm{F}_{2}$ plants from the $\mathrm{B} 73 \times \mathrm{Mol} 7$ hybrids. We intended to use oligo-FISH to cytologically characterize the multiple COs on chromosome 10, which were delineated by genotype and genome sequence data ${ }^{30,31}$.

We re-analyzed the chromosome 10 genotyping data of the IBMRIL population (see Methods section) and chose 10 IBMRILs for oligo-FISH analysis. A total of 45 genetically immortalized CO events were identified across the 10 IBMRILs using genotypic data sets ${ }^{30,31}$ (Supplementary Table 1). The genotypic data indicated that these 10 IBMRILs contain multiple COs located throughout chromosome 10 (Fig. 6). Several of these IBMRILs contain putative COs located in the pericentromeric regions. FISH using the hapB and hapM probes revealed that nearly all of the recombination events predicted by the genotypic data were associated with a visible CO. For example, line Mo189 was predicted to contain a proximal B73 chromosome segment, including the centromere, and a distal B73 segment on the long arm (Fig. 6, Supplementary Data 3). Oligo-FISH mapping revealed that chromosome 10 of Mo189 contains two chromosomal segments, including the centromere, from B73 and two segments from Mo17 (Figs. 6 and 7a, b). Similarly, both genotyping and oligo-FISH data showed that chromosome 10 of Mo270 contains two segments from Mo17, including the centromere, and two segments from B73 (Figs. 6 and 7c, d). However, we were not able to detect the putative small chromosomal fragments, which were indicated by a single or few DNA markers, such as those associated with lines Mo270 and Mo346 (Fig. 6). These regions may represent small chromosomal segments from B73 or Mo17, but cannot be visualized by oligoFISH using the hapB and hapM probes.

COs close to the centromere of chromosome 10 were detected in several IBMRILs. For example, an EP on chromosome 10 of Mo189 was mapped at FLA 68.8 on the long arm (Figs. 6 and $7 \mathrm{~b}$ ). The EP that is most close to the centromere was mapped at FLA 84.6 on the long arm of chromosome 10 of Mo029 (Fig. 6). Marker analysis on 209 IBMRILs showed that 11 additional lines (in addition to the five lines in Fig. 6) contain a CO with a breakpoint at the same position as Mo189 or more close to the centromere of chromosome 10 . Therefore, the intermating process is highly effective to recover and accumulate the rare $\mathrm{CO}$ events occurred in the pericentromeric regions. 

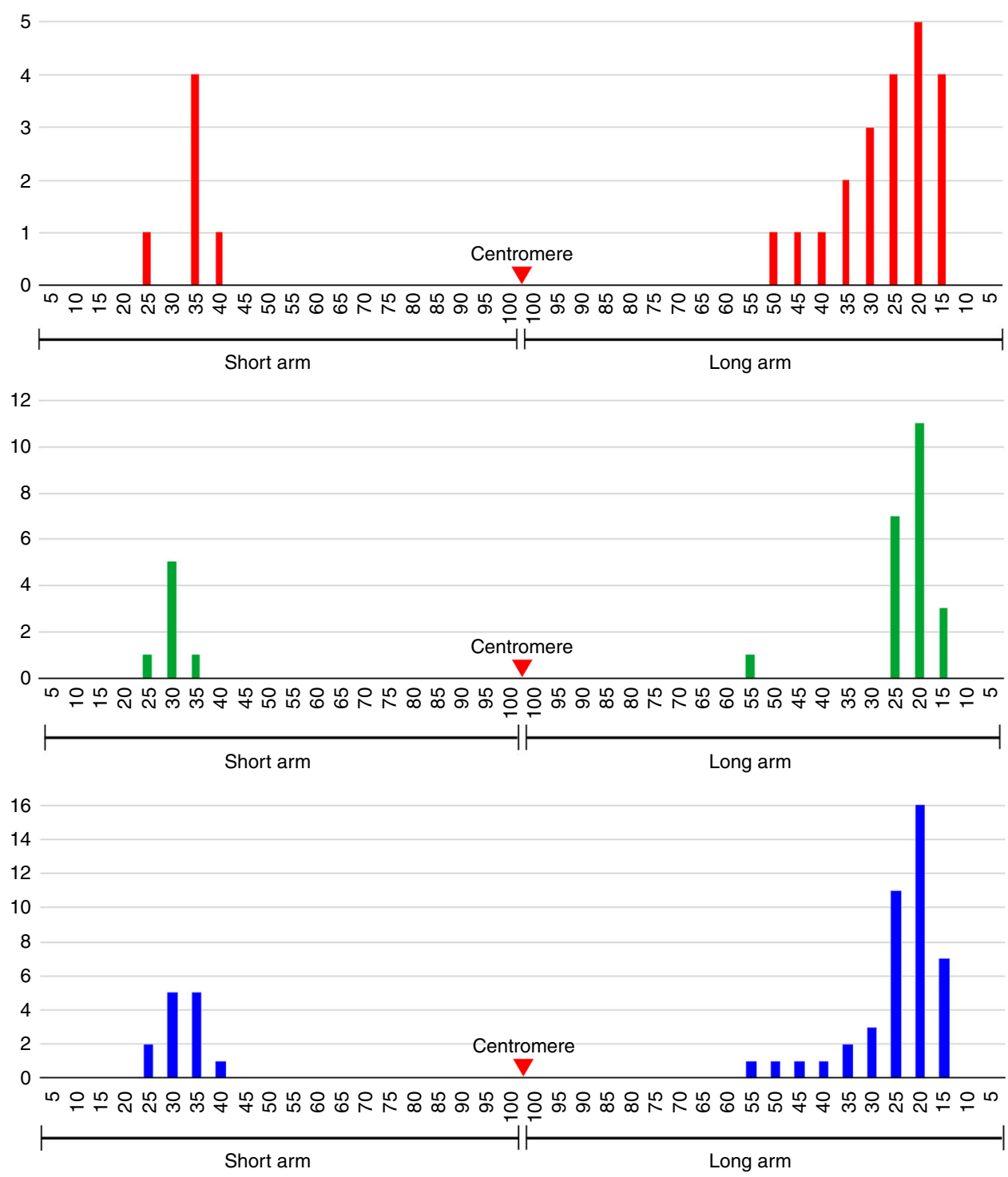

Fig. 4 Distribution of chromosomal exchange positions on chromosome 10. Upper panel, middle panel, and lower panel show exchange positions (EPs) on chromosome 10 of B73, Mo17, and combined chromosome 10, respectively. Each interval on the $x$-axes represents $5 \%$ of the length of the short or long arm. $y$-axes indicate the number of EPs within a particular $5 \%$ of the short arm or long arm. The red triangles point to the centromere position on the chromosome

\section{Discussion}

COs are not evenly distributed along the chromosomes in most plant species studied so far. Suppression of genetic recombination in the pericentromeric regions has been well documented in a number of plant species $8,9,13$. Physical mapping of a large number of RNs indicated the lack of recombination in the pericentromeric regions of maize chromosomes, although such analysis was conducted only in a single inbred line $\mathrm{KYS}^{32}$. The RN-based analysis showed that the pericentromeric regions, which account approximately half of the length of each chromosome, were nearly free of $\mathrm{RNs}^{32}$. Recombination suppression in the pericentromeric regions was also supported by physical mapping of genetically achored DNA markers on maize chromosomes ${ }^{33-35}$. We demonstrate that several IBMRILs contain COs close to the centromere of chromosome 10 (Fig. 6), which were not detected in the $58 \mathrm{~F}_{2}$ plants (Fig. 4). Thus, the intermating process was effective to accumulate $\mathrm{COs}$ in recombination-suppressed chromosomal regions, which can be used to generate favorable allelic combinations of genes residing in these regions.
If a single $\mathrm{CO}$ is associated with chromosome 10 during meiosis of the $\mathrm{B} 73 \times$ Mo17 hybrid, two of the four chromatids will be involved in this CO event. Therefore, $50 \%$ of the progeny chromosome 10 will become a recombinant chromosome that may be detected by FISH. Our FISH analysis of $58 \mathrm{~F}_{2} \mathrm{BM}$ plants indicated that 60 of the 116 copies of chromosome 10 are not recombinant chromosomes (Supplementary Data 2). We sequenced $10 \mathrm{~F}_{2} \mathrm{BM}$ plants, including 13 copies of chromosome 10 that were FISH negative (Fig. 5). Sequence analysis showed that $69 \%(9 / 13)$ of these FISH-negative chromosome 10 were confirmed as non-recombinant chromosomes. Therefore, the percentage of chromosome 10 without $\mathrm{CO}$ is estimated to be $36 \%(69 \% \times 60 / 116)$ in the $F_{2}$ population. Luo et al. $(2019)^{36}$ have recently developed a technique to sequence individual gametophyte using a maize hybrid derived from a cross between inbreds Zheng58 and SK. They reported that $18 \%$ male gametes and $31 \%$ female gametes from this hybrid do not contain a $\mathrm{CO}$ on chromosome $10^{36}$, which would result in $25 \%$ of chromosome 10 without $\mathrm{CO}$ in $\mathrm{F}_{2}$ population. Thus, our data 

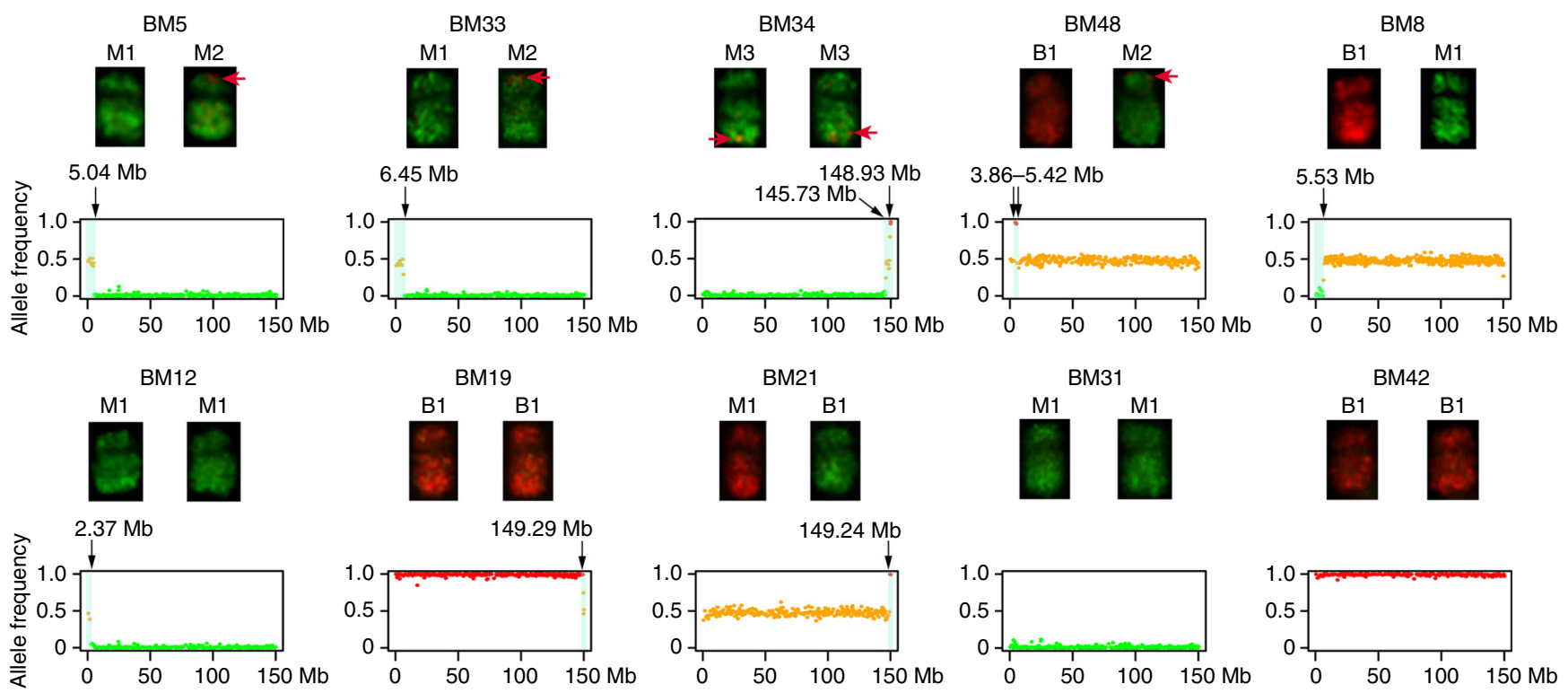

Fig. 5 Oligo-FISH and sequencing analyses of $10 \mathrm{~F}_{2}$ plants. Red arrows indicate the B73 FISH signals located on Mo17-derived chromosome 10 in BM5, BM33, BM34, and BM48. These crossovers (COs) were inconsistently detected in different metaphase cells in each line. Black arrows mark the chromosomal exchange position on chromosome 10. No CO was detected in any of the chromosome 10 in BM31 and BM42. The $y$-axis of each sequencing profile represents the allele frequency of B73 (red) or Mo17 (green). The $x$-axis represents the sequence position of chromosome 10 (150.98 Mb). Orange dots indicate the mix of red (B73 allele) and green (Mo17 allele) dots

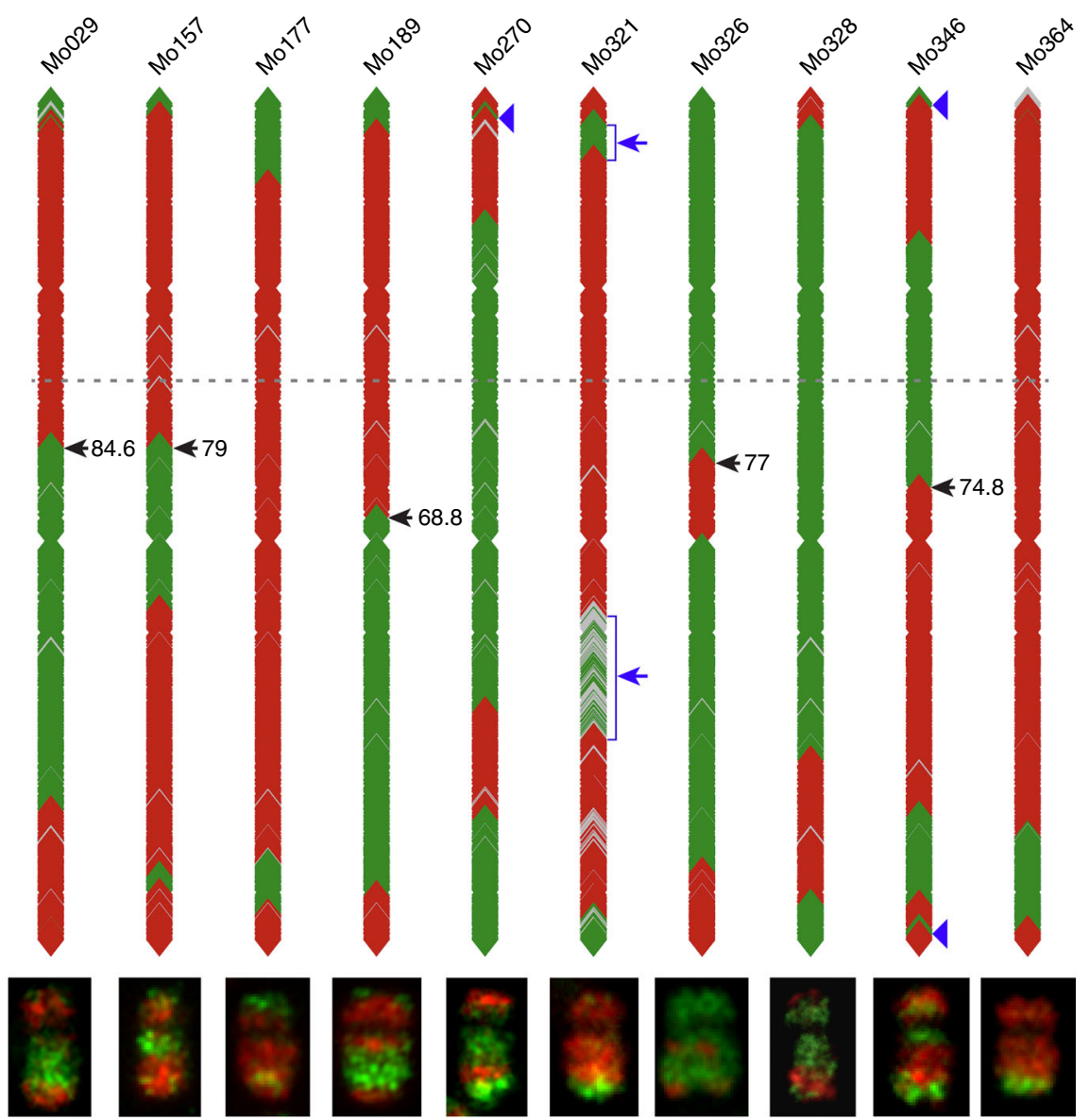

Fig. 6 Schematic illustration of genotyping data and oligo-FISH results of 10 lines from the IBMRIL population. The genotyping data is illustrated by location of markers from B73 (red) and Mo17 (green), respectively. Gray indicates missing genotyping data. A dashed line marks the putative position of the centromere on each of the marker-based diagrams. The regions indicated by blue arrows on Mo321 were not consistently visualized by oligo-FISH. Blue arrowheads on Mo270 and Mo346 point to small regions that were not identified by oligo-FISH. Black arrows point to the chromosomal exchange positions (EPs) and their fractional length of the arm (FLA), all on the long arm 

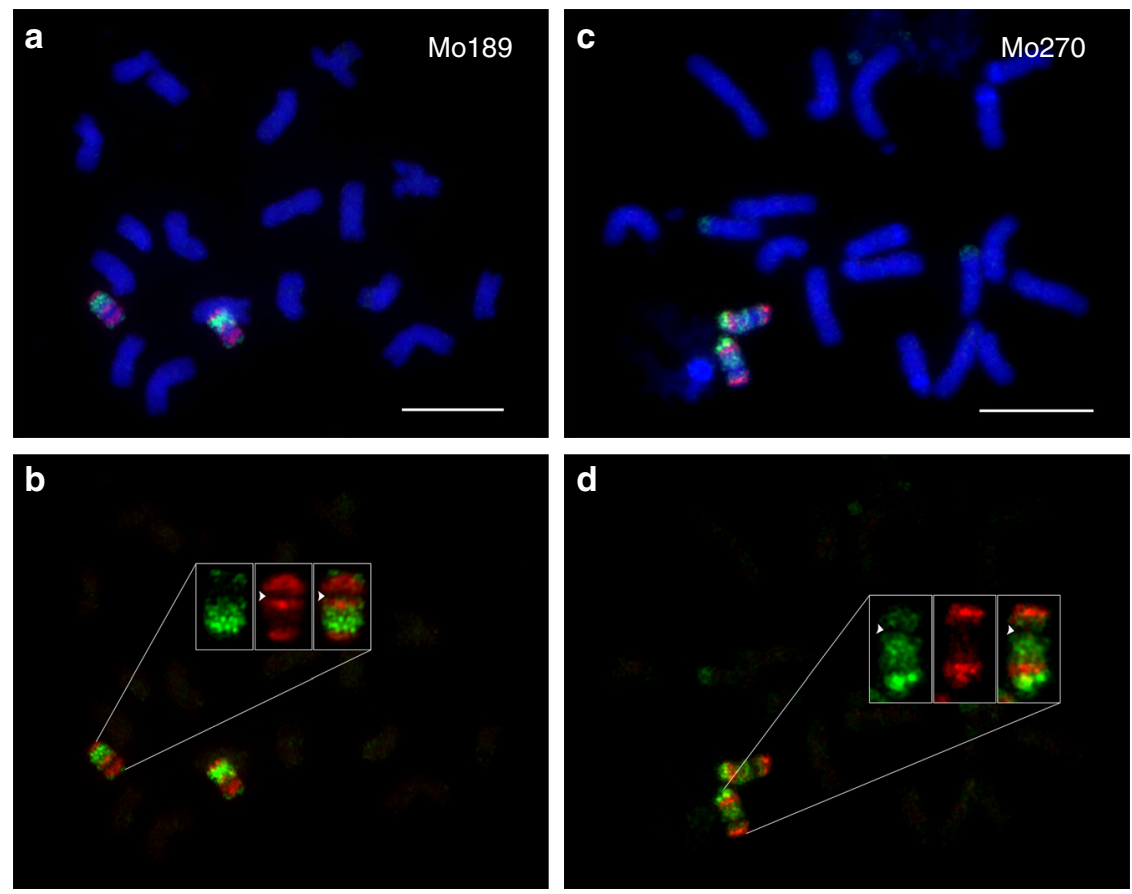

Fig. 7 Oligo-FISH characterization of two lines from the IBMRIL population. a Oligo-FISH analysis of Mo189. Bar $=10 \mu \mathrm{m}$. $\mathbf{b}$ The oligo-FISH signals of the two chromosome 10 were digitally separated from panel (a). Signals from one copy of chromosome 10 are exemplified. Two B73 chromosomal blocks (red), including the centromere, and two Mo17 blocks (green) are identified on chromosome 10. Arrowheads indicate the centromeric region that shows weak signals due to few oligos available. c Oligo-FISH mapping of Mo270. Bar $=10 \mu \mathrm{m}$. $\mathbf{d}$ The oligo-FISH signals of the two chromosome 10 were digitally separated from panel (c). Signals from one copy of chromosome 10 are exemplified. Two Mo17 blocks (green), including the centromere and two B73 blocks (red) are identified on chromosome 10. Arrowheads indicate the centromeric region that shows weak signals due to few oligos available. The original gray-scale images used to generate the color images in Fig. 7a and c are provided as a Source Data file

of $36 \%$ chromosome 10 without $\mathrm{CO}$ in the $\mathrm{B} 73 \times \mathrm{Mo17} \mathrm{F}_{2}$ population is higher than the predicted $25 \%$ in the Zheng $58 \times$ $\mathrm{SK}_{2}$ population. This variation may be caused by the different maize inbreds used in the $\mathrm{F}_{1}$ hybrids. In Arabidopsis, meiotic COs on chromosome 4 , the smallest chromosome, were analyzed by genotyping $\mathrm{F}_{2}$ plants resulting from a cross between ecotypes Columbia and Landsberg ${ }^{37}$. Approximately 30\% (423 of 1404) chromosome 4 do not contain a $\mathrm{CO}^{37}$, which is close to $36 \%$ of non-recombinant maize chromosome 10 in our study.

A major recent advance of FISH is the application of probes based on synthetic oligos ${ }^{38-40}$. Oligo-based chromosome painting probes have been developed in several plant species and applied in various types of chromosomal studies ${ }^{41-47}$. We demonstrate that haplotype-specific oligo-FISH can be to visualize meiotic COs derived from homologous chromosomes derived from different maize inbreds. Although COs can be mapped using RNs or protein markers ${ }^{7,32}$, these traditional methods can only predict the positions of COs on SCs in a specific maize line. In contrast, haplotype-specific FISH maps the chromosomal breakpoints derived from historical COs, including breakpoints derived from multiple COs on recombinant inbred lines from the intermated B73 $\times$ Mo17 population (Fig. 7 ), which can not be analyzed by the traditional methods. The haplotype-specific FISH probes will have unique applications in tracking specific chromosomes derived from a single genotype (Supplementary Note 1). For example, such probes can be used to examine the extent of somatic recombination reported in several plant species ${ }^{48,49}$. Haplotype-specific probes can potentially be used to distinguish true homologous chromosome pairing from pairing of homoeologous chromosome with minor structural variation in polyploid species.

\section{Methods}

Plant materials and associated genomic data sets. Hybrids between B73 and Mo17 were developed and sibling $F_{1}$ plants were pollinated to generate $F_{2}$ seeds. IBMRIL seed was originally obtained from the Maize Genetics Cooperative Stock Center and was bulked and quality-controlled as described ${ }^{50}$. This ensured correspondence with the genotype data used to construct the original IBM2 map ${ }^{30}$, which are available at MaizeGDB ${ }^{51}$. To add genetic detail for the chromosome 10 investigation, data for 1761 SNPs genotyped for the IBMRILs ${ }^{31}$ was merged with the 64 markers available in the IBM2:chromosome 10 data set. Physical genomic coordinates of the markers corresponding to the $\mathrm{Zm00001d}$ genome ${ }^{24}$ were obtained using resources available at MaizeGDB ${ }^{51}$.

\section{Development of haplotype-specific oligo-FISH probes. Chorus software} (https://github.com/forrestzhang/Chorus) was used to generate single copy 45-nt oligos from chromosome 10 of $\mathrm{B}^{24} 3^{24}$ and $\mathrm{Mo} 17^{23}$, respectively, with a parameter of Chorus "-1 45 -homology 75 -step 5". B73 reference genome Zm-B73-REFERENCE-GRAMENE-4.024 and Mol7 genome Zm-Mo17-REFERENCE-CAU-1.0 ${ }^{23}$ were download from NCBI under project PRJNA10769 and PRJNA358298, respectively. To validate the repetitiveness of each oligo, we generated the frequency distribution of 17-mers from B73 (SRR407544 and SRR407504, JGI) and Mo17 (SRR5826129, ${ }^{23}$ ) genomic sequencing data. Any 17-mers with a frequency more than 100 in the genome was defined as a repetitive 17-mer. Oligos containing 2 or more repetitive 17 -mers were discarded.

In total, 175,437 and 174,728 oligos from B73 and Mo17 chromosome 10 were generated, respectively (Supplementary Note 1). To identify oligos that distinguish the chromosome 10 sequences from the two inbreds, we mapped B73 (Mo17) oligos to Mo17 (B73) reference genome using BWA ALN ${ }^{52}$ with default parameters and blastn in BLAST ${ }^{53}$ with parameter "-task blastn". B73 (Mo17) oligos that were not identified on Mo17 (B73) chromosome 10 were defined as PAV oligos. B73 (Mo17) oligos containing mismatches and/or indels to Mol7 (B73) chromosome 10 were defined as SNP probe. We only retained SNP oligos with mismatches and/ or indels located between 10 and 35 bp within each oligo. For each B73 oligo, the B73 sequences at SNPs and/or indels were replaced by the Mo17 sequences, and vice versa. Therefore, SNP oligos are in pairs, in which one oligo set is homologous to the $\mathrm{B} 73$ genome but showed mismatches and/or indels to Mol7 genome and vice versa. We then calculated the $\Delta \mathrm{Tm}$ (difference of melting temperature) between $\mathrm{B} 73$ oligo and Mo17 oligo of each oligo pair using primer $3^{25,26}$. We discarded oligo pairs with $\Delta \mathrm{Tm}>5{ }^{\circ} \mathrm{C}$ to avoid hybridization bias toward one 
variant. The SNP oligo pairs were divided into four different classes: oligos with 1 SNP, 2 SNPs, 3-4 SNPs, and 5 or more SNPs. The two probes used for CO characterization, hapB and hapM, included all PAV oligos and oligos with 3-4 SNPs, and 5 or more SNPs. These two probes contained 14,498 and 13,753 oligos, respectively (Supplementary Data 4 ).

Oligo-FISH. All seeds were germinated in the laboratory and plants were transferred to the greenhouse. Root tips were collected from the plants and were treated with nitrous oxide at a pressure of $160 \mathrm{psi}(\sim 10.9 \mathrm{~atm})$ for $2 \mathrm{~h}$. Root tips were harvested from plants growing in greenhouses. Chromosome preparation from root tips followed published protocols ${ }^{42}$. We synthesized 10 different oligo pools (five for B73, five for Mo17), including two PAV oligo pools, and four paired (i.e., eight total) oligo pools with 1 SNP, 2 SNPs, 3-4 SNPs, or $\geq 5$ SNPs. The B73 oligo probes were labeled with digoxigenin and Mo17 probes were labeled with biotin. Amplification and labeling of the oligo-based probes were according to published protocols ${ }^{41}$. All biotin-labeled probes were detected by anti-biotin fluorescein (Vector Laboratories, Burlingame, California) and digoxigenin-labeled probes were detected by antidigoxigenin rhodamine (Roche Diagnostics, Indianapolis, Indiana). DAPI (4',6diamidino-2-phenylindole) was used to counterstain chromosomes in the VectaShield antifade solution (Vector Laboratories). FISH images were captured using a QImaging Retiga EXi Fast 1394 CCD. The original gray scale images were processed with Meta Imaging Series 7.5 software. The contrast of the gray scale images was adjusted and merged using Adobe Photoshop CS3 software.

For each unambiguously identified meiotic $\mathrm{CO}$ we measured the distance, or length $(\mu \mathrm{m})$, from the chromosomal exchange point (EP) to the telomere of the respective chromosome arm. The length $(\mu \mathrm{m})$ of the respective chromosome arm bearing the $\mathrm{CO}$ was also measured. The chromosomal position of each EP is presented as a FLA (Fractional Length of the Arm) by dividing the measured distance by the total length of the chromosome arm. DRAWID (https://doi.org/10.3897/ compcytogen.v1li4.20830) was used to measure the distance from the FISH signal to the telomere of the corresponding arm, as well as the length of the entire arm.

Sequencing and analysis. Genomic DNA was isolated from $10 \mathrm{~F}_{2}$ plants (BM5, BM33, BM34, BM48, BM8, BM12, BM19, BM21, BM31, BM42) for Illumina sequencing. We generated an average of $2.41 \times$ coverage of $150 \mathrm{bp}$ pair-end sequences from these plants. The sequence data was mapped to the B73 RefGen_v4 ${ }^{24}$ by BWA MEM software with default parameters ${ }^{52}$. Reads with mapping quality more than 50 were retained for SNP calling. SNPs were detected using freebayes with parameter "-C $1-\mathrm{m} 50-\mathrm{q} 30^{\text {" } 54}$. Raw SNPs were further filtered using vcffilter in freebayes package by "DP $<40$ " and "QUAL/DP $>10$ ". The bin map was constructed using a sliding window approach ${ }^{55}$ with minor modification. SNPs were scanned in 300-SNP-windows and the genotype of each window defined by the percentage of B73 and Mo17 SNPs: the windows were called homozygous $\mathrm{B} 73$ genotype when $\mathrm{SNP}_{\mathrm{B} 73}$ is more than $80 \%$ in the window, homozygous Mo17 genotype when $\mathrm{SNP}_{\mathrm{Mo1}}$ is more than $80 \%$ in the window and heterozygous genotype when $\mathrm{SNP}_{\mathrm{B} 73}$ was between 20 and $80 \%$.

Reporting summary. Further information on research design is available in the Nature Research Reporting Summary linked to this article.

\section{Data availability}

Data supporting the findings of this work are available within the paper and its Supplementary Information files. A reporting summary for this article is available as a Supplementary Information file. The datasets generated and analyzed during the current study are available from the corresponding author upon request. Genomic sequencing data of the 10 maize lines have been deposited to NCBI Sequence Read Archive (SRA) under project PRJNA540894 (https://www.ncbi.nlm.nih.gov/bioproject/? term $=$ PRJNA540894). The source data underlying Figs. 1, 2b, 3a, 3b, 7a, and $c$ are provided as a Source Data file.

Received: 27 April 2019; Accepted: 20 September 2019; Published online: 10 October 2019

\section{References}

1. Keeney, S., Giroux, C. N. \& Kleckner, N. Meiosis-specific DNA double-strand breaks are catalyzed by Spo11, a member of a widely conserved protein family. Cell 88, 375-384 (1997).

2. Gray, S. \& Cohen, P. E. Control of meiotic crossovers: from double-strand break formation to designation. Annu. Rev. Genet. 50, 175-210 (2016).

3. Jones, G. H. \& Franklin, F. C. H. Meiotic crossing-over: obligation and interference. Cell 126, 246-248 (2006).

4. Wang, S. X., Zickler, D., Kleckner, N. \& Zhang, L. R. Meiotic crossover patterns: obligatory crossover, interference and homeostasis in a single process. Cell Cycle 14, 305-314 (2015).
5. Zickler, D. \& Kleckner, N. Recombination, pairing, and synapsis of homologs during meiosis. Cold Spring Harb. Persp. Biol. 7, a016626 (2015).

6. Carpenter, A. T. C. Electron microscopy of meiosis in Drosophila melanogaster females: II: the recombination nodule - a recombination-associated structure at pachytene. Proc. Natl Acad. Sci. USA 72, 3186-3189 (1975).

7. Lhuissier, F. G. P., Offenberg, H. H., Wittich, P. E., Vischer, N. O. E. \& Heyting, C. The mismatch repair protein MLH1 marks a subset of strongly interfering crossovers in tomato. Plant Cell 19, 862-876 (2007).

8. Gill, K. S., Gill, B. S., Endo, T. R. \& Taylor, T. Identification and high-density mapping of gene-rich regions in chromosome group 1 of wheat. Genetics 144, 1883-1891 (1996).

9. Kunzel, G., Korzun, L. \& Meister, A. Cytologically integrated physical restriction fragment length polymorphism maps for the barley genome based on translocation breakpoints. Genetics 154, 397-412 (2000).

10. Cheng, Z., Presting, G. G., Buell, C. R., Wing, R. A. \& Jiang, J. M. Highresolution pachytene chromosome mapping of bacterial artificial chromosomes anchored by genetic markers reveals the centromere location and the distribution of genetic recombination along chromosome 10 of rice. Genetics 157, 1749-1757 (2001)

11. Lamb, J. C. et al. Single-gene detection and karyotyping using small-target fluorescence in situ hybridization on maize somatic chromosomes. Genetics 175, 1047-1058 (2007).

12. Danilova, T. V. \& Birchler, J. A. Integrated cytogenetic map of mitotic metaphase chromosome 9 of maize: resolution, sensitivity, and banding paint development. Chromosoma 117, 345-356 (2008).

13. Iovene, M., Wielgus, S. M., Simon, P. W., Buell, C. R. \& Jiang, J. M. Chromatin structure and physical mapping of chromosome 6 of potato and comparative analyses with tomato. Genetics 180, 1307-1317 (2008).

14. Tang, X. M. et al. Cross-species bacterial artificial chromosome-fluorescence in situ hybridization painting of the tomato and potato chromosome 6 reveals undescribed chromosomal rearrangements. Genetics 180, 1319-1328 (2008).

15. King, J. et al. Physical and genetic mapping in the grasses Lolium perenne and Festuca pratensis. Genetics 161, 315-324 (2002).

16. Khrustaleva, L. I., de Melo, P. E., van Heusden, A. W. \& Kik, C. The integration of recombination and physical maps in a large-genome monocot using haploid genome analysis in a trihybrid Allium population. Genetics $\mathbf{1 6 9}$, 1673-1685 (2005).

17. Zhang, W. et al. Meiotic homoeologous recombination-based mapping of wheat chromosome 2B and its homoeologues in Aegilops speltoides and Thinopyrum elongatum. Theor. Appl. Genet. 131, 2381-2395 (2018).

18. Mikel, M. A. \& Dudley, J. W. Evolution of North American dent corn from public to proprietary germplasm. Crop Sci. 46, 1193-1205 (2006).

19. Swanson-Wagner, R. A. et al. All possible modes of gene action are observed in a global comparison of gene expression in a maize F1 hybrid and its inbred parents. Proc. Natl Acad. Sci. USA 103, 6805-6810 (2006).

20. Stupar, R. M. \& Springer, N. M. Cis-transcriptional variation in maize inbred lines B73 and Mo17 leads to additive expression patterns in the F1 hybrid. Genetics 173, 2199-2210 (2006).

21. Swanson-Wagner, R. A. et al. Paternal dominance of trans-eQTL influences gene expression patterns in maize hybrids. Science 326, 1118-1120 (2009).

22. Schnable, P. S. et al. The B73 maize genome: complexity, diversity, and dynamics. Science 326, 1112-1115 (2009).

23. Sun, S. L. et al. Extensive intraspecific gene order and gene structural variations between Mo17 and other maize genomes. Nat. Genet. 50, 1289-1295 (2018)

24. Jiao, Y. P. et al. Improved maize reference genome with single-molecule technologies. Nature 546, 524-527 (2017).

25. Koressaar, T. \& Remm, M. Enhancements and modifications of primer design program Primer3. Bioinformatics 23, 1289-1291 (2007).

26. Untergasser, A. et al. Primer3-new capabilities and interfaces. Nucleic Acids Res. 40, e115 (2012)

27. Zhao, H. N. et al. Gene expression and chromatin modifications associated with maize centromeres. G3 6, 183-192 (2016).

28. Lee, M. et al. Expanding the genetic map of maize with the intermated $B 73 \mathrm{x}$ Mo17 (IBM) population. Plant Mol. Biol. 48, 453-461 (2002).

29. Zhang, N. Y. et al. Fine quantitative trait loci mapping of carbon and nitrogen metabolism enzyme activities and seedling biomass in the maize IBM mapping population. Plant Physiol. 154, 1753-1765 7 (2010).

30. Sharopova, N. et al. Development and mapping of SSR markers for maize. Plant Mol. Biol. 48, 463-481 (2002).

31. Ganal, M. W. et al. A large maize (Zea mays L.) SNP genotyping array: development and germplasm genotyping, and genetic mapping to compare with the B73 reference genome. PLoS ONE 6, e28334 (2011).

32. Anderson, L. K. et al. High-resolution crossover maps for each bivalent of Zea mays using recombination nodules. Genetics 165, 849-865 (2003).

33. Koumbaris, G. L. \& Bass, H. W. A new single-locus cytogenetic mapping system for maize (Zea mays L.): overcoming FISH detection limits with marker-selected sorghum (S. propinquum L.) BAC clones. Plant J. 35, 647-659 (2003). 
34. Wang, C. J. R., Harper, L. \& Cande, W. Z. High-resolution single-copy gene fluorescence in situ hybridization and its use in the construction of a cytogenetic map of maize chromosome 9. Plant Cell 18, 529-544 (2006).

35. Figueroa, D. M. \& Bass, H. W. Development of pachytene FISH maps for six maize chromosomes and their integration with other maize maps for insights into genome structure variation. Chromosome Res. 20, 363-380 (2012).

36. Luo, C., Li, X., Zhang, Q. H. \& Yan, J. B. Single gametophyte sequencing reveals that crossover events differ between sexes in maize. Nat. Commun. 10, 785 (2019)

37. Drouaud, J. et al. Variation in crossing-over rates across chromosome 4 of Arabidopsis thaliana reveals the presence of meiotic recombination "hot spots". Genome Res. 16, 106-114 (2006).

38. Beliveau, B. J. et al. Versatile design and synthesis platform for visualizing genomes with Oligopaint FISH probes. Proc. Natl Acad. Sci. USA 109, 21301-21306 (2012).

39. Beliveau, B. J. et al. Single-molecule super-resolution imaging of chromosomes and in situ haplotype visualization using Oligopaint FISH probes. Nat. Commun. 6, 7147 (2015).

40. Jiang, J. M. Fluorescence in situ hybridization in plants: recent developments and future applications. Chromosome Res. 27, 153-165 (2019).

41. Han, Y. H., Zhang, T., Thammapichai, P., Weng, Y. Q. \& Jiang, J. M. Chromosome-specific painting in cucumis species using bulked oligonucleotides. Genetics 200, 771-779 (2015)

42. Braz, G. T. et al. Comparative Oligo-FISH mapping: an efficient and powerful methodology to reveal karyotypic and chromosomal evolution. Genetics 208, 513-523 (2018).

43. He, L., Braz, G. T., Torres, G. A. \& Jiang, J. M. Chromosome painting in meiosis reveals pairing of specific chromosomes in polyploid Solanum species. Chromosoma 127, 505-513 (2018).

44. Hou, L. L. et al. Chromosome painting and its applications in cultivated and wild rice. BMC Plant Biol. 18, 110 (2018).

45. Meng, Z. et al. Comprehensively characterizing the cytological features of Saccharum spontaneum by the development of a complete set of chromosome-specific oligo probes. Front. Plant Sci. 9, 1624 (2018).

46. Xin, H. et al. Chromosome painting and comparative physical mapping of the sex chromosomes in Populus tomentosa and Populus deltoides. Chromosoma 127, 313-321 (2018).

47. Albert, P. S. et al. Whole-chromosome paints in maize reveal rearrangements, nuclear domains, and chromosomal relationships. Proc. Natl Acad. Sci. USA 116, 1679-1685 (2019).

48. Vig, B. K. Somatic crossing over in Glycine max (L) Merrill: effect of some inhibitors of DNA synthesis on induction of somatic crossing over and point mutations. Genetics 73, 583-596 (1973).

49. Carlson, P. S. Mitotic crossing-over in a higher plant. Genet. Res. 24, 109-112 (1974).

50. Lauter, N., Moscou, M. J., Habiger, J. \& Moose, S. P. Quantitative genetic dissection of shoot architecture traits in maize: Towards a functional genomics approach. Plant Genome 1, 99-110 (2008).

51. Andorf, C. M. et al. MaizeGDB update: new tools, data and interface for the maize model organism database. Nucleic Acids Res. 44, D1195-D1201 (2016).

52. Li, H. \& Durbin, R. Fast and accurate short read alignment with BurrowsWheeler transform. Bioinformatics 25, 1754-1760 (2009).

53. Altschul, S. F. et al. Gapped BLAST and PSI-BLAST: a new generation of protein database search programs. Nucleic Acids Res. 25, 3389-3402 (1997).

54. Garrison, E. \& Marth, G. Haplotype-based variant detection from short-read sequencing. Preprint at https://arxiv.org/abs/1207.3907 (2012).
55. Song, W. B. et al. Genetic dissection of maize seedling root system architecture traits using an ultra-high density bin-map and a recombinant inbred line population. J. Integr. Plant Biol. 58, 266-279 (2016).

\section{Acknowledgements}

We thank the Maize Genetics Cooperative Stock Center for the original provision of seed stocks and MaizeGDB for genetic and genomic data curation. We thank Kyle King and Colton McNinch for bioinformatics efforts to attach physical genome coordinates to the genetic markers used in this study. F.Y. and Z.D. are supported by grant 31571730 from the National Natural Science Foundation of China and from the National Education Ministry, Key Laboratory of Plant Genetic Improvement and Comprehensive Utilization, Fujian Agriculture and Forestry University. This research is supported by NSF grant IOS1444514 and MSU startup funds to J.J.

\section{Author contributions}

J.J. conceived the project; L.d.V.M., F.Y., and H.W. conducted the oligo-FISH experiments; H.Z. designed the oligo-FISH probes; T.D. and N.L. analyzed the genotyping data of the IBMRILs; K.S. and J.M.-R. synthesized probes and provided reagents; Z.D., A.T. and J.A.B. developed and provided the resources; H.Z., J.A.B., and J.J. wrote the paper.

\section{Competing interests}

K.S. and J.M.-R. are employed by Arbor Biosciences. J.M.-R. has personal financial interest in Arbor Biosciences. The remaining authors declare no competing interests.

\section{Additional information}

Supplementary information is available for this paper at https://doi.org/10.1038/s41467019-12646-z.

Correspondence and requests for materials should be addressed to J.J.

Peer review information Nature Communications thanks Trude Schwarzacher, Jianbing Yan and the other, anonymous, reviewer(s) for their contribution to the peer review of this work. Peer reviewer reports are available.

Reprints and permission information is available at http://www.nature.com/reprints

Publisher's note Springer Nature remains neutral with regard to jurisdictional claims in published maps and institutional affiliations.

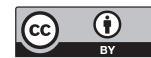

Open Access This article is licensed under a Creative Commons Attribution 4.0 International License, which permits use, sharing, adaptation, distribution and reproduction in any medium or format, as long as you give appropriate credit to the original author(s) and the source, provide a link to the Creative Commons license, and indicate if changes were made. The images or other third party material in this article are included in the article's Creative Commons license, unles indicated otherwise in a credit line to the material. If material is not included in the article's Creative Commons license and your intended use is not permitted by statutory regulation or exceeds the permitted use, you will need to obtain permission directly from the copyright holder. To view a copy of this license, visit http://creativecommons.org/ licenses/by/4.0/.

(C) The Author(s) 2019 